\title{
Active Cushing syndrome patients have increased ectopic fat deposition and bone marrow fat content compared to cured patients and healthy subjects: a pilot ${ }^{1} \mathrm{H}-\mathrm{MRS}$ study
}

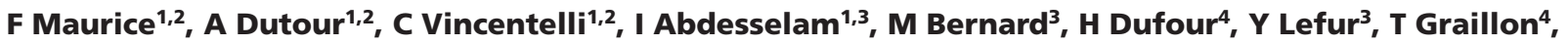 \\ F Kober ${ }^{3}$, P Cristofari', E Jouve ${ }^{6}$, L Pini ${ }^{3}$, R Fernandez ${ }^{7}$, C Chagnaud7, T Brue $^{8,9}$, F Castinetti ${ }^{8,9}$ and B Gaborit ${ }^{1,2}$ \\ ${ }^{1}$ Aix Marseille Univ, INSERM, INRA, C2VN, Marseille, France, ²Department of Endocrinology, Pôle ENDO, APHM, Marseille, France, \\ ${ }^{3}$ Aix Marseille Univ, CNRS, CRMBM, Marseille, France, ${ }^{4}$ Department of Neurosurgery, APHM, CHU Timone, Marseille, \\ France, ${ }^{5}$ Columbia University, New York, New York, USA, ${ }^{6}$ Medical Evaluation Department, Assistance-Publique \\ Hôpitaux de Marseille, CIC-CPCET, Marseille, France, ${ }^{7}$ Radiology Department, Conception Hospital, Marseille, France, \\ ${ }^{8}$ Aix-Marseille Univ, Institut National de la Santé et de la Recherche Médicale (INSERM), U1251, Marseille Medical \\ Genetics (MMG), Marseille, France, and ${ }^{9}$ Department of Endocrinology, Assistance Publique - Hôpitaux de Marseille \\ (AP-HM), Hôpital de la Conception, Centre de Référence des Maladies Rares Hypophysaires HYPO, Marseille, France \\ Correspondence \\ should be addressed \\ to B Gaborit \\ Email \\ benedicte.gaborit@ap-hm.fr
}

\begin{abstract}
Objective: Glucocorticoid excess is one of the most important causes of bone disorders. Bone marrow fat (BMF) has been identified as a new mediator of bone metabolism. Cushing syndrome (CS) is a main regulator of adipose tissue distribution but its impact on BMF is unknown. The objective of the study was to evaluate the effect of chronic hypercortisolism on BMF.

Design: This was a cross-sectional study. Seventeen active and 17 cured ACTH-dependent CS patients along with 17 controls (matched with the active group for age and sex) were included.

Methods: The BMF content of the femoral neck and $\mathrm{L} 3$ vertebrae were measured by ${ }^{1} \mathrm{H}-\mathrm{MRS}$ on a 3-Tesla wide-bore magnet. Bone mineral density (BMD) was evaluated in patients using dual-energy $\mathrm{X}$-ray absorptiometry.

Results: Active CS patients had higher BMF content both in the femur $(82.5 \pm 2.6 \%)$ and vertebrae $(70.1 \pm 5.1 \%)$ compared to the controls $(70.8 \pm 3.6 \%, P=0.013$ and $49.0 \pm 3.7 \% P=0.005$, respectively). In cured CS patients (average remission time of 43 months), BMF content was not different from controls at both sites $(72.3 \pm 2.9 \%$ (femur) and $46.7 \% \pm 5.3 \%$ (L3)). BMF content was positively correlated with age, fasting plasma glucose, HbA1c, triglycerides and visceral adipose tissue in the whole cohort and negatively correlated with BMD values in the CS patients.

Conclusions: Accumulation of BMF is induced by hypercortisolism. In remission patients, BMF reached values of controls. Further studies are needed to determine whether this increase in marrow adiposity in CS is associated with bone loss.
\end{abstract}

\section{Introduction}

Chronic glucocorticoid (GC) excess is the most frequent etiology of secondary bone loss (1). It induces severe bone loss via multiple and incompletely understood
(C) 2018 European Society of Endocrinology Printed in Great Britain mechanisms (2). Indeed, GC has direct effects on bone. They impair the replication, differentiation and functions of osteoblasts, induce apoptosis of mature osteoblasts 
and osteocytes and promote osteoclastogenesis. GC also have indirect effects that lead to bone loss through interference with both calcium metabolism and secretion of hormones, such as growth hormone and sex steroids. Cushing's syndrome (CS) is an interesting clinical model of endogenous GC excess. Compared to patients treated with GC, there are no confounding factors due to the underlying primary disease. CS induces major bone disorder, which is an important factor among various disease comorbidities (3). Identifying patients with increased fracture risk is challenging because of the great inter-individual variability in bone defects and because dual-energy X-ray absorptiometry (DXA) measurements are not sufficient to predict fracture risk. Furthermore, after correction of hypercortisolism, a discordance between bone mineral density (BMD) and the risk of fracture was evidenced $(4,5)$.

Bone marrow fat (BMF), which is a unique and dynamic fat depot, has been suggested to play a pivotal role in bone metabolism (6). Sharing the same precursor in the bone marrow, mesenchymal stromal cells, osteoblasts and adipocytes have a reciprocal relationship in differentiation and function that drives bone integrity. Many studies have found that changes in BMF volume correlate with reduced BMD as well as a history of low-energy fractures in patients with osteoporosis or aging $(7,8)$. Increased BMF was also found in patients with metabolic diseases, such as obesity $(9,10)$, diabetes mellitus (11) and anorexia nervosa (12).

Recent developments in MRI with ${ }^{1} \mathrm{H}$-spectroscopy ( $\left.{ }^{1} \mathrm{H}-\mathrm{MRS}\right)$ allow for direct and sensitive non-invasive quantification of BMF. GCs are known to modulate adipose tissue proliferation and differentiation as well as induce a specific redistribution of body fat. However, the impact of GC on BMF is unclear. Only one study (13) evaluated BMF changes with MRI using manual segmentation of fat after remission of Cushing's disease and showed discordant results with a modulation of pelvic BMF but no modulation of total BMF.

Therefore, the purpose of this study was to evaluate $\mathrm{BMF}$ by ${ }^{1} \mathrm{H}$-MRS in patients in remission (at least 2 years) vs active ACTH-dependent CS in comparison with ageand sex-matched controls.

\section{Subjects and methods}

\section{Patients}

Patients with ACTH-dependent CS were prospectively recruited from December 2014 to June 2017 in the Endocrinology and in the Neurosurgery Departments of
Marseille University Hospital, France. The active group consisted of newly diagnosed patients or patients with uncontrolled disease despite multiple treatments. The remission group included patients who were in remission for at least 2 years, but no more than 6 years, regardless of their treatment modality.

Exclusion criteria included an age below 18 years, pregnancy or breastfeeding, presence of another chronic disease known to affect bone density (renal failure, rheumatoid arthritis, hyperthyroidism, primary hyperparathyroidism, alcoholism, malabsorption syndromes, chronic liver or obstructive pulmonary disease, premature ovarian failure, prolonged immobilization, ...) and contraindications for MRI.

ACTH-dependent CS was diagnosed according to the usual clinical and biological criteria, including (1) elevated 24-h urinary free cortisol (UFC) for at least two times; (2) increase of basal plasma cortisol levels with a loss of the circadian pattern and inappropriate unsuppressed plasma ACTH levels; (3) lack of cortisol suppression after a low-dose dexamethasone test $(0.5 \mathrm{mg}$ every $6 \mathrm{~h}$ for $48 \mathrm{~h}$ ). Patients were considered to be in remission after treatment if they presented either with secondary adrenal insufficiency based on the following criteria: low morning plasma cortisol levels after $24 \mathrm{~h}$ of withdrawal of hydrocortisone or with eucortisolism based on the following criteria: (1) Twenty-four-hour UFC below or within the normal range for at least two times; (2) basal plasma cortisol levels below or within the normal range with a restoration of physiological circadian pattern and appropriate plasma ACTH levels; (3) cortisol suppression after the overnight $1 \mathrm{mg}$ dexamethasone test or if they were controlled with anti-cortisolic therapy based on the following criteria: 24-h UFC below or within the normal range at every control during follow-up for at least 2 years.

\section{Control subjects}

Control subjects were recruited through advertisements and matched with active patients for age and sex. Exclusion criteria included an age below 18 years, pregnancy or breastfeeding, contraindication for MRI, presence of a chronic disease known to affect bone density as for patients, past or actual chronic treatment with GC or other medication for osteoporosis (bisphosphonates, raloxifene, calcitonin or PTH), metabolic syndrome and a BMI above $25 \mathrm{~kg} / \mathrm{m}^{2}$.

All the patients and volunteers gave their written informed consent, and the study protocol was approved by the local ethics committee (Marseille, Comité de Protection 
des Personnes, Sud-Méditerranée II identification 2014-A01302-45 and 2016-A00026-45, clinical trial identification: NCT02335996 and NCT02848703).

\section{Study protocol}

\section{Clinical assessments}

BMI was calculated for each participant by dividing weight in kilograms by height in squared meters. We recorded a history of medical conditions for all subjects as well as past and current medications and the duration of uncontrolled CS, which was defined as the period of time between symptoms onset (as determined by the patients) and inclusion in the study for patients with active CS. For the patients in remission, duration of uncontrolled disease was defined as the period of time between symptoms onset and remission of hypercortisolism after treatment.

\section{Biochemical evaluation}

Fasting blood tests for glucose, total cholesterol, LDL cholesterol, HDL cholesterol, serum triglyceride, HbA1c, 25-hydroxyvitamin D, calcium, parathyroid hormone and serum C-terminal telopeptide of type I collagen (CTX-1) were taken in all the patients the day of inclusion. Control subjects were asked to perform a blood test with a fasting blood glucose and lipid unless they had one from less than a year. The results were determined with standard analytical methods. Twentyfour-hour UFC excretion was quantified using a specific radioimmunoassay (reference: IM1841, Beckman Coulter Diagnostics). Plasma cortisol levels and plasma ACTH levels were measured with an electrochemiluminescent immunoassay (Roche Diagnostics). CTX-1 was quantified using ELISA assays (cross laps).

\section{${ }^{1} \mathrm{H}-\mathrm{MRS}$ spectroscopy}

All MRI acquisitions were carried out in a 3-Tesla widebore magnet (Magnetom Verio, Siemens Healthineers, Erlangen, Germany) equipped with a 32-element phased array coil. The participants were scanned in a head-first supine position. A spectroscopic volume of interest was positioned using a multi-slice FLASH image series with three different orientations (axial, coronal and sagittal), and a point-resolved single-voxel proton spectroscopy (PRESS) sequence was used to determine the molecular content of lipids and water. The parameters of PRESS ${ }^{1} \mathrm{H}$ MRS were echo time $=35 \mathrm{~ms}$, repetition time $=1000 \mathrm{~ms}$ and receiver bandwidth $=2000 \mathrm{~Hz}$. Non-water-suppressed spectra (16 averages) were acquired to measure fat content. Full-relaxed spectra (1 excitation) were acquired to measure water content. At the lumbar spine, the voxel (voxel size $15 \times 15 \times 15 \mathrm{~mm}^{3}$ ) was placed within the L3 vertebral body, whereas at the right hip, the voxel (voxel size $10 \times 10 \times 10 \mathrm{~mm}^{3}$ ) was positioned within the femoral neck (Supplementary Fig. 1, see section on supplementary data given at the end of this article). Spectral data were analyzed after phase, baseline and frequency shift corrections using a semi-automatic method (AMARES, Advanced Method for Accurate, Robust and Efficient Spectral fitting) implemented under an Interactive Data Language (IDL) environment (Exelis Visual Information Solutions, Inc., Boulder, Colorado, USA). Four peaks could be isolated in bone marrow spectra: unsaturated lipids (UL corresponding to olefinic protons, double bond $\mathrm{CH}=\mathrm{CH}-$ ) at $5.3 \mathrm{ppm}$, water $(\mathrm{W})$ at $4.65 \mathrm{ppm}$, residual lipids (RL corresponding to methylene protons, $\alpha$ - to a double bond $-\mathrm{CH}=\mathrm{CHCH}_{2}-$ ) at $2.03 \mathrm{ppm}$ and saturated lipids (SL corresponding to bulk methylene protons, $-\mathrm{CH}_{2}-$ ) at $1.3 \mathrm{ppm}$ (14). We added the peaks fitted at $1.3 \mathrm{ppm}$ and $2.03 \mathrm{ppm}$ to represent one fat peak. It was decided not to include UL in the measurement because it was not always well resolved. The calculations were performed as follows: BMF content $(\%)=I$ fat $/($ Ifat $+I$ water $) \times 100$, where $I$ is the peak area under the curve.

One patient in the remission group had a hip prosthesis and therefore did not undergo ${ }^{1} \mathrm{H}$-MRS at the femur.

To evaluate interscan variability, ${ }^{1} \mathrm{H}-\mathrm{MRS}$ was performed in five volunteers before and after repositioning. The average interscan coefficient of variation $(\mathrm{CV})$ of BMF was $1.93 \%$ and $3.55 \%$ for vertebrae and femur, respectively.

All femoral spectra were of good quality, while vertebral spectra in four cases had shimming problems and were unsuitable for analysis.

\section{MRI assessment of abdominal adiposity}

A 3D three-point Dixon volume interpolated breathhold gradient echo sequence (VIBE) was used to acquire fat and water images of the volume between vertebrae L4 and the sacrum. The parameters of the Dixon sequence were $\mathrm{TE}=2.45 / 3.67 \mathrm{~ms}$, $\mathrm{TR}=6.56 \mathrm{~ms}$, receiver bandwidth $=530 / 574 \mathrm{~Hz}, \mathrm{FOV}=450 \mathrm{~mm}$, matrix size (in $3 \mathrm{D})=225 \times 448$. Areas of abdominal adipose tissue (AT) compartments were measured with a semi-automatic method at a single axial slice at the intervertebral disc L4L5 using in-house software running under IDL (15). Fascia 
between superficial and deep subcutaneous AT was traced manually. Total subcutaneous AT (TSCAT) was calculated by adding superficial subcutaneous AT (sSCAT) and deep subcutaneous AT (dSCAT). The mean intra-subject CV between five repeated measurements was $3.6 \%$ for visceral AT (VAT), $4.3 \%$ for dSCAT and $4.8 \%$ for sSCAT. The mean inter-subject CV between two observers of 45 patients was 9.6\% for VAT, $7.8 \%$ for dSCAT and $8.7 \%$ for sSCAT.

\section{Dual-energy $X$-ray absorptiometry}

The patients underwent DXA to measure the BMD of the whole body, the right hip and the spine (L1-L4) using a GE Lunar iDXA (GE Healthcare).The CV is $0.41 \%$ for lumbar spine and $1.36 \%$ for femoral neck (16).

\section{Statistical analysis}

All statistics were performed using Prism (v 7.01) software (GraphPad Software Inc.). All quantitative results are presented as the means \pm standard error of mean (S.E.M.) or median (25th, 75th percentile) when the distribution was non-Gaussian. All qualitative results are presented as numbers and percentages. A $P$ value less than $5 \%$ was considered to be statistically significant. All qualitative datasets were compared using Fisher's test. All normally distributed quantitative datasets were compared using Student's $t$-test, and non-normally distributed quantitative datasets were compared using Mann-Whitney. Analyses of covariance (ANCOVA) were performed using patients' group and sex as fixed effect, age, VAT and HbA1c as covariate. Least-square means were calculated (adjusted means) and pairwise comparisons between groups where $P$ value adjusted using Bonferroni method. Pearson's (Gaussian distribution) or Spearman's (non-Gaussian distribution) correlations were used to evaluate associations between BMF and anthropometrical, biological and bone density parameters. A multiple linear regression analysis was performed with Statview version 5.0 among the variables correlated at the linear correlation. An ANCOVA model under JASP version 0.8.2 was used to adjust BMF to covariates (age, sex, VAT and BMD values).

\section{Results}

\section{Clinical and biological characteristics}

Seventeen patients in each group completed the study (Fig. 1). The three groups did not differ in age or sex (Table 1). Subjects had a mean age of 51.6 $\pm 3.4,48.3 \pm 3.0$ and $50.3 \pm 2.1$ years in the active, remission and control groups, respectively. Patients from both the active and remission groups did not differ in duration of uncontrolled disease, and all had comparable plasma midnight ACTH, cortisol levels and 24-h UFC at diagnosis. Fifteen patients of the active and remission group had Cushing's disease and two had an ectopic source of ACTH. Patients with ectopic CS had shorter duration of uncontrolled CS compared to patients with Cushing disease: 9.5 (2.513.5) months vs $25(12-48)$ months, $P=0.043$. Thirteen patients of the active group had newly diagnosed CS, whereas four were uncontrolled despite multiple treatments, and they did not differ for main clinical characteristics. In the remission group, the mean duration of remission was $43 \pm 4$ months, and the mean duration of hydrocortisone supplementation was $39 \pm 4$ months. Among the 17 patients in the remission group, 13 had glucocorticoid deficiency at the time of the MRI with a mean hydrocortisone dose of $26.5 \pm 1.3 \mathrm{mg}$ /day (full range: 20 to $30 \mathrm{mg} /$ day). One treatment modality was sufficient to achieve remission for 11 patients, whereas for the others, two to three treatments were necessary. The three groups did not differ in terms of estrogen deficiency, hormonal replacement therapy (estrogen and/ or progestogen) and number of fragility fractures. Patients in the active and the remission groups did not differ with regards to anti-osteoporotic therapy or testosterone deficiency, which was correctly substituted in each case. Current BMI (i.e BMI at the time of inclusion in the study) did not differ between the active $\left(27.11 \pm 0.87 \mathrm{~kg} / \mathrm{m}^{2}\right)$ and remission $\left(26.62 \pm 1.22 \mathrm{~kg} / \mathrm{m}^{2}\right)$ groups. BMI at diagnosis (i.e BMI at discovery of CS) was also comparable between the two groups. As expected, controls had lower BMI, $22.14 \pm 0.71 \mathrm{~kg} / \mathrm{m}^{2}$, compared to patients $(P<0.001)$. Active patients had higher fasting blood glucose levels and serum triglyceride levels than controls. Patients in the remission group had lower HbA1c and significantly lower fasting blood glucose than their active counterparts. Serum triglyceride levels displayed a tendency to be lower in the remission group compared to the active group, and they were not different from the controls.

\section{Abdominal AT distribution}

The mean AT areas in each abdominal compartment are shown in Table 2. Active patients had a pathological distribution of abdominal AT with higher VAT and dSCAT/sSCAT ratios than controls $(P<0.001$ and $P=0.013$ respectively). Patients in the remission group had decreased VAT compared to active patients, but VAT 

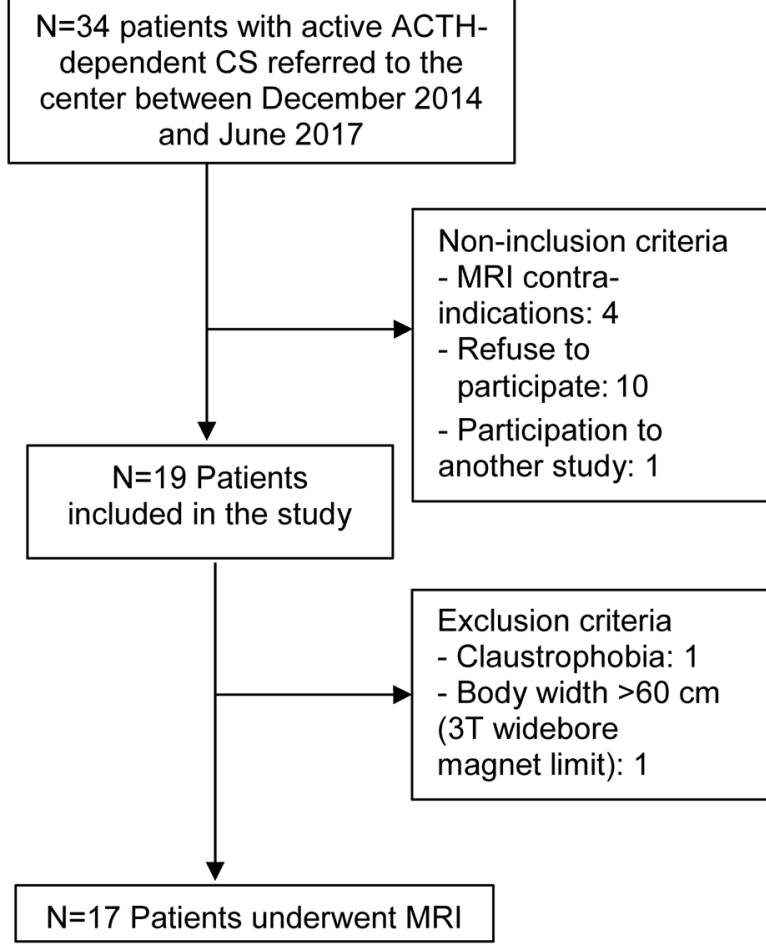

\section{Figure 1}

Flow chart of the study. CS, Cushing syndrome.

remained higher than that in controls $(P<0.05)$. dSCAT/ sSCAT ratio was significantly lower in remission group compared to the active group and was statistically not different from controls.

\section{BMF content and BMD}

Active patients had higher BMF content (Fig. 2) in their femur $(82.5 \pm 2.6 \%)$ and in L3 $(70.1 \pm 5.1 \%)$ than the controls $(70.8 \pm 3.6 \%, P=0.013$ and $49.0 \pm 3.7 \%, P=0.005$, respectively). In the remission group, BMF content was not different from controls at both locations $(72.3 \pm 2.9 \%$ and $46.7 \% \pm 5.3 \%$, respectively). After adjustment to VAT, both BMF content in femurs and vertebrae were still significantly higher in the active group compared to the remission group ( $P=0.015$ and $P=0.040$, respectively). Further adjustment for age, sex and HbA1c showed that active patients remained with higher BMF at both locations compared to remission patients (Table 3). Exclusion of the five patients with anti-osteoporotic therapy (two in the active and three in the remission group) did not statistically change our results (For BMF at femur: $P=0.008$ between active and controls groups, $P=0.019$ between active and remission groups. For BMF at vertebrae: $P=0.005$ between active and controls groups,

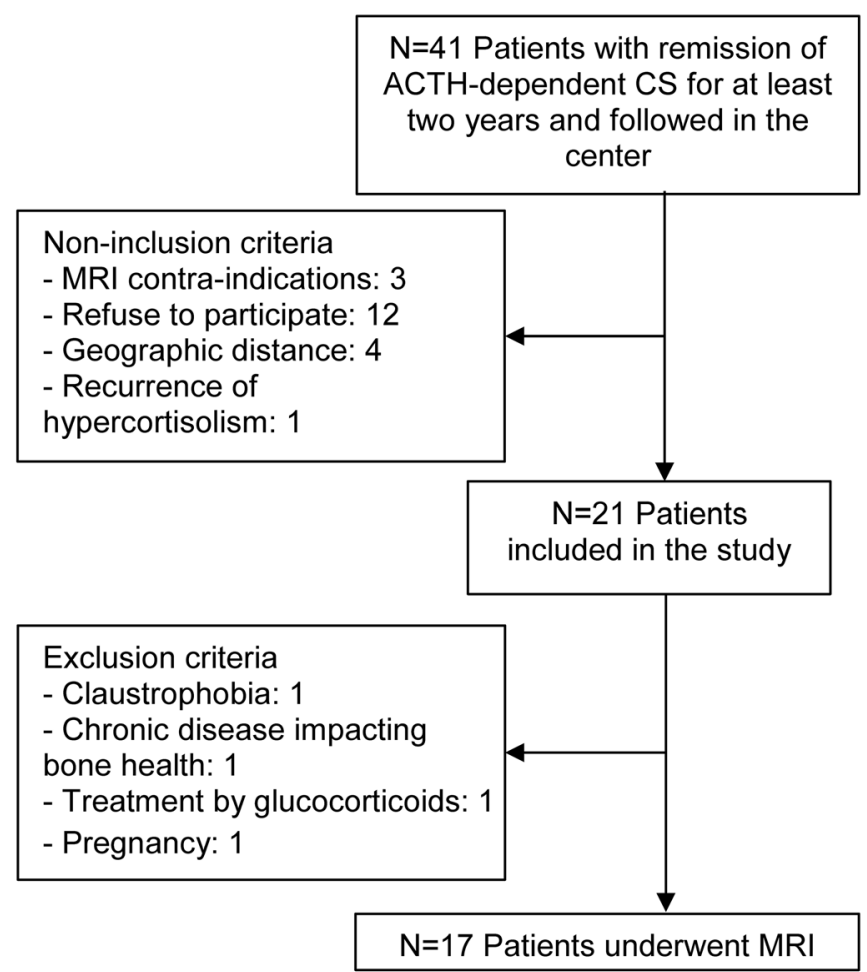

$P=0.010$ between active and remission groups). Remission and controls groups did not differ at either location.

Osteodensitometric parameters (Table 4) did not differ significantly between active and remission groups. Two patients of each group (11.8\%) were considered osteoporotic. Seven from the active group (41.2\%) and six from remission group (35.3\%) were considered osteopenic according to WHO criteria using T-score (17).

\section{Correlation analysis}

BMF content in L3 and femurs were positively correlated $(r=0.53 P=0.002)$ and correlated with age (Supplementary Table 1) but not with BMI. No correlation was found with the duration of uncontrolled CS, duration of hydrocortisone supplementation or UFC levels. Both femoral and L3 BMF correlated positively with $\mathrm{HbA}_{1} \mathrm{C}$ but only L3 BMF correlated additionally with fasting blood glucose levels $(r=0.42, P=0.042)$. L3 BMF also correlated with serum triglyceride levels and with VAT, whereas femoral BMF correlated only with the dSCAT/ SSCAT ratio. CTX-1 was positively correlated with BMF (at femur: $r=0.42, P=0.018$ ), but not with BMD (at the same location: $r=-0.29, P=0.156)$. In subgroup analyses, BMF remained correlated with BMD values only in the 
Table 1 Clinical and biological characteristics of the study participants. Data presented as $n(\%)$ or mean \pm S.E.M. or median (25th percentile, 75th percentile).

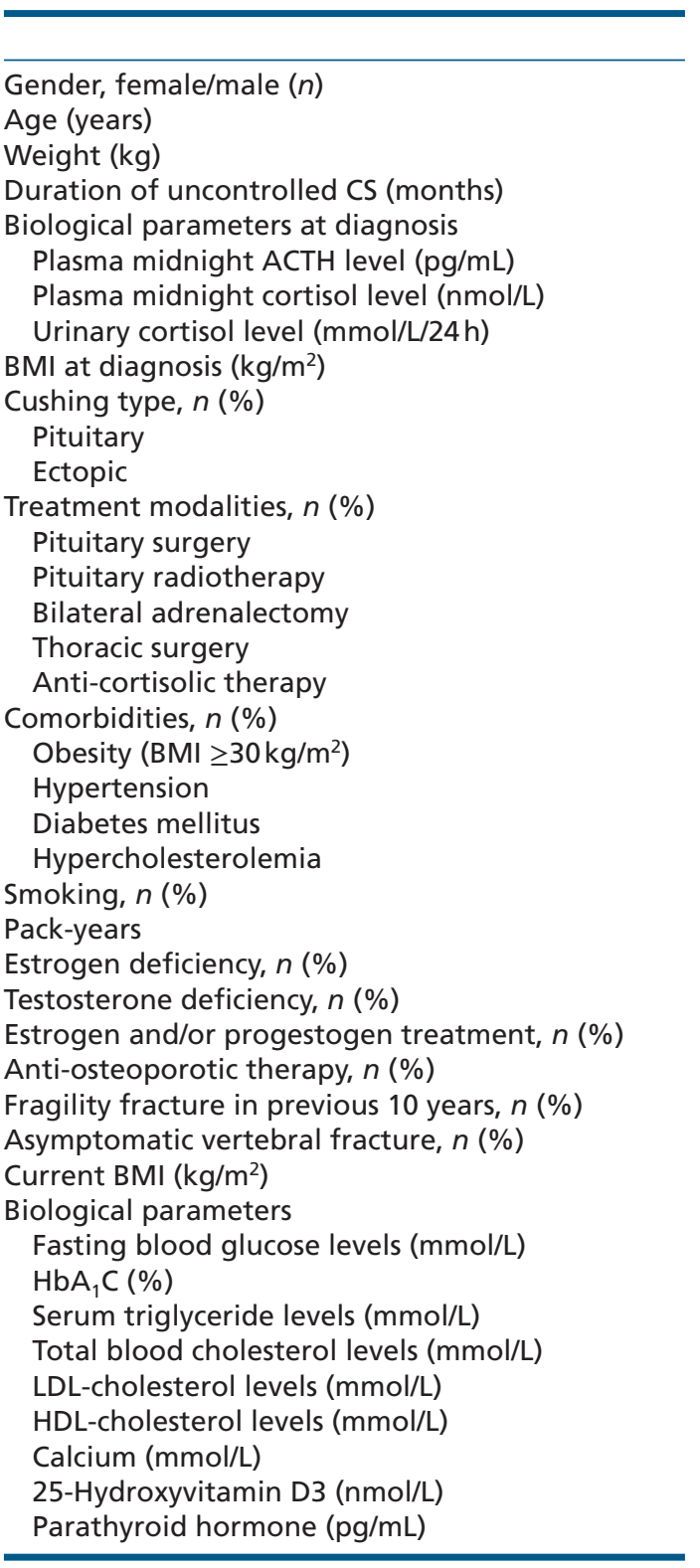

\begin{tabular}{c} 
Active group $(n=17)$ \\
\hline $12 / 5$ \\
$51.6 \pm 3.4$ \\
$74 \pm 3.0$ \\
$25.3 \pm 4.3$ \\
$50(33-103)$ \\
$499.5(298.5-657.5)$ \\
$1260(270-2130)$ \\
$27.11 \pm 0.87$ \\
$15(88.2)$ \\
$2(11.8)$ \\
$4(23.5)$ \\
$2(11.8)$ \\
0 \\
0 \\
$2(11.8)$ \\
$3(17.6)$ \\
$12(70.6)$ \\
$7(41.2)$ \\
$6(35.3)$ \\
$5(29.4)$ \\
$40(11-45)$ \\
$7(58.3)$ \\
$3(60)$ \\
$3(25)$ \\
$2(11.8)$ \\
$1(5.9)$ \\
$5(29.6)$ \\
$27.11 \pm 0.87$ \\
$5.70 \pm 0.32$ \\
$6.82 \pm 0.51$ \\
$1.66 \pm 0.25$ \\
$5.58 \pm 0.28$ \\
$3.32 \pm 0.24$ \\
$1.54 \pm 0.13$ \\
$2.29 \pm 0.09$ \\
$53 \pm 20$ \\
$47 \pm 13$ \\
\\
\\
\\
\end{tabular}

\begin{tabular}{c}
\hline Remission group $(n=17)$ \\
\hline $13 / 4$ \\
$48.3 \pm 3.0$ \\
$71.6 \pm 3.3$ \\
$33.5 \pm 8.0$ \\
$64(35-90)$ \\
$498(335.0-699.5)$ \\
$1012(462-3629)$ \\
$29.6 \pm 1.97$ \\
$15(88.2)$ \\
$2(11.8)$ \\
$15(88.2)$ \\
$1(5.6)$ \\
$8(44.4)$ \\
0 \\
$1(5.6)$ \\
$5(29.4)$ \\
$4(23.5)$ \\
$2(11.8)$ \\
$2(11.8)$ \\
$3(17.6)$ \\
$5(2-5)$ \\
$7(58.3)$ \\
$2(50)$ \\
$0(0)$ \\
$3(17.6)$ \\
$3(17.6)$ \\
$4(23.5)$ \\
$26.62 \pm 1.22$ \\
\\
\\
\end{tabular}

\begin{tabular}{clc}
\hline Controls $(n=17)$ & & $P$ value \\
\cline { 1 - 1 } $13 / 4$ & & NS \\
$50.3 \pm 2.1$ & & NS \\
$62.6 \pm 2.7$ & & $<0.05^{b, c}$ \\
- & & NS
\end{tabular}

NS

NS

NS

NS

NS

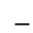

$\begin{array}{cc} & \text { NS } \\ - & <0.05^{\mathrm{a}} \\ & \text { NS } \\ 4(23.5) & \mathrm{NS} \\ 10(7-30) & \mathrm{NS} \\ 5(29.4) & \mathrm{N} .05^{\mathrm{a}} \\ - & \mathrm{NS} \\ 2(15.4) & \mathrm{NS} \\ 0(0) & \mathrm{NS} \\ 1(5.9) & \mathrm{NS} \\ - & \mathrm{NS} \\ 22.14 \pm 0.71 & <0.05^{\mathrm{b}, \mathrm{c}}\end{array}$

Normal ranges: Fasting blood glucose: $4.11-5.89 \mathrm{mmol} / \mathrm{L}$; Serum triglyceride $<2.26 \mathrm{mmol} / \mathrm{L} ;$ Total blood cholesterol $<6.20 \mathrm{mmol} / \mathrm{L} ; \mathrm{HDL}-\mathrm{cholesterol}$ $>1.20 \mathrm{mmol} / \mathrm{L} ;$ Calcium: 2.15-2.50 mmol/L; 25-hydroxyvitamin D: 75-250 nmol/L; Parathydroid hormone: $15-65 \mathrm{pg} / \mathrm{mL}$.

${ }^{\text {a }} P<0.05$ between active and remission groups; ${ }^{\mathrm{b}} P<0.05$ between active group and controls; ${ }^{\mathrm{c}} P<0.05$ between remission group and controls. NS, not significant.

remission group (Supplementary Table 1). Neither HbA1c nor VAT was correlated with BMF or BMD values in the active group or in the remission group. However, L3 BMF was correlated with VAT in controls (Pearson correlation $r=0.66, P=0.007)$, and there was a trend with femur BMF $(r=0.42, P=0.09)$.

After performing a multiple regression analysis including L3 BMF as a dependent variable (Table 5) and VAT, age and sex as independent variables, BMF was positively associated with VAT $(\beta=0.37 ; P=0.030)$ and age $(\beta=0.42 ; P=0.012)$.

There was an inverse correlation between femoral BMF and BMD at the same location, and there was a similar connection between L3 BMF and total body BMD. After performing a multiple regression analysis that includes femoral BMF as a dependent variable and BMD at the same location, age and sex (known to be related to BMF content) as independent variables, femoral BMF was negatively 
Table 2 Abdominal adipose tissue compartments. Data presented as mean \pm S.E.M.

\begin{tabular}{|c|c|c|c|c|}
\hline & Active group & Remission group & Controls & $P$ value \\
\hline VAT $\left(\mathrm{cm}^{2}\right)$ & $147.0 \pm 15.4$ & $100.5 \pm 12.0$ & $64.4 \pm 6.6$ & $<0.05^{\mathrm{a}, \mathrm{b}, \mathrm{c}}$ \\
\hline TSCAT $\left(\mathrm{cm}^{2}\right)$ & $295.7 \pm 27.6$ & $301.2 \pm 35.3$ & $172.7 \pm 26.0$ & $<0.05^{b, c}$ \\
\hline sSCAT $\left(\mathrm{cm}^{2}\right)$ & $131.1 \pm 11.9$ & $158.8 \pm 24.3$ & $86.2 \pm 13.4$ & $<0.05^{b, c}$ \\
\hline VAT/sSCAT ratio & $1.29 \pm 0.19$ & $0.80 \pm 0.14$ & $0.89 \pm 0.11$ & $<0.05^{a}$ \\
\hline $\mathrm{dSCAT}\left(\mathrm{cm}^{2}\right)$ & $164.5 \pm 17.7$ & $142.3 \pm 17.0$ & $86.5 \pm 14.8$ & $<0.05^{b, c}$ \\
\hline dSCAT/sSCAT ratio & $1.27 \pm 0.09$ & $1.08 \pm 0.14$ & $1.00 \pm 0.12$ & $<0.05^{b}$ \\
\hline
\end{tabular}

${ }^{\text {a }} P<0.05$ between active and remission groups; ${ }^{\mathrm{b}} P<0.05$ between active group and controls; ${ }^{\mathrm{c}} P<0.05$ between remission group and controls. dSCAT, deep subcutaneous adipose tissue; sSCAT, superficial subcutaneous adipose tissue; TSCAT, total subcutaneous adipose tissue; VAT, visceral adipose tissue.

associated with $\operatorname{BMD}(\beta=-0.51 ; P=0.003)$ and positively associated with age $(\beta=0.38 ; P=0.018$ with $\mathrm{BMD})$.

After performing a multiple regression analysis with L3 BMF as a dependent variable and total body BMD, age and sex as independent variables, BMF content was negatively associated with total body $\operatorname{BMD}(\beta=-0.99$; $P=0.003)$. However, there were no correlations with age.

\section{Discussion}

We have demonstrated for the first time with ${ }^{1} \mathrm{H}$-MRS that patients with active ACTH-dependent CS have increased BMF content in the right femoral neck and in the lumbar spine compared to age- and sex-matched normal-weight controls. Furthermore, in cured ACTH-dependent CS patients we found that BMF content was similar to controls at either site after 43 months of remission.

Our work confirms and follows results from previous preliminary studies on exogenous glucocorticoids, including one study that involved pre-menopausal women treated with GC for lupus that showed with MRI that there was a high percentage of marrow to fat conversion in the proximal femur (18). However, inflammatory connective tissue pathology could directly influence bone marrow adipocytes. Furthermore, inflammatory diseases are known to be associated with bone loss.

Small sample sized studies did not find significant variation of marrow adiposity in CS patients vs healthy volunteers using dual-energy CT scanning (19). However, only four patients with Cushing disease were included in this study. Besides, CT is limited by the long acquisition times, high radiation dose and is related to the linear attenuation coefficient. Single-voxel proton magnetic resonance spectroscopy $\left({ }^{1} \mathrm{H}-\mathrm{MRS}\right)$ remains the gold standard for the quantification of marrow AT in humans (20).

The study of Geer et al. (13) found a decrease in pelvic BMF after 20.1 months of remission but not when total
BMF was considered. However, their study design had no control patients and used quantification methods that are not widely used. Our results showed a significant difference between active and remission patients with regards to both femoral neck and L3 BMF; furthermore, we found that $\mathrm{L} 3 \mathrm{BMF}$ and osteodensitometric parameters were negatively associated, even after controlling for age and sex. However, osteodensitometric parameters were not significantly different between active and remission patients. Moreover, in subgroup analyses, BMF remained correlated with BMD values only in the remission group, confirming that BMD is not a surrogate marker for BMF. This could be explained by the remission time being too short. Indeed, a study by Kristo et al. (21) found a significant increase in BMD measurements only after 71 months of remission and not earlier. Therefore, our results showed discordance between variation of BMF with a significant decrease in remission patients and a lack of BMD value variation. Data of epidemiological studies found that fracture risk waned rapidly after remission in patients with CS (22) as well as after stopping glucocorticoid treatment in a large study including patients with exogenous hypercortisolism (23). BMF content seems to better reflect this brief risk improvement after remission than BMD values. Furthermore, in hypercortisolism secondary
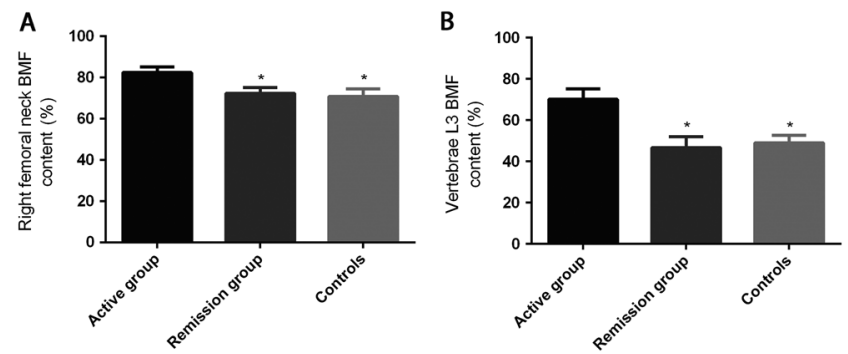

Figure 2

Bone marrow fat content measured with ${ }^{1} \mathrm{H}$-MRS at right femoral neck (A) and at vertebrae L3 (B). Data presented as mean \pm S.E.M. ${ }^{*} P<0.05$ compared to active patients. 
Table 3 Adjusted means with 95\% confidence interval of bone marrow fat content. $P$ for the difference between groups was calculated using an ANCOVA model, pairwise comparisons were adjusted using Bonferroni method.

\begin{tabular}{|c|c|c|c|c|}
\hline & Active group & Remission group & Controls & $\boldsymbol{P}$ \\
\hline \multicolumn{5}{|l|}{ L3 BMF } \\
\hline Adjusted for age and sex & $70.3 \pm 4.8^{b}$ & $39.4 \pm 4.4^{a}$ & $50.2 \pm 3.2$ & $<0.0001$ \\
\hline Adjusted for age sex and $\mathrm{HbA} 1 \mathrm{c}$ & $80.0 \pm 11.7$ & $38.9 \pm 4.2^{\mathrm{a}}$ & $47.9 \pm 4.5$ & 0.003 \\
\hline Adjusted for age sex and VAT & $65.6 \pm 6.3$ & $38.4 \pm 4.4^{\mathrm{a}}$ & $51.8 \pm 3.5$ & 0.002 \\
\hline \multicolumn{5}{|l|}{ Femur BMF } \\
\hline Adjusted for age and sex & $84.9 \pm 2.7$ & $73.1 \pm 2.9^{a}$ & $75.6 \pm 2.9$ & 0.01 \\
\hline Adjusted for age, sex and $\mathrm{HbA} 1 \mathrm{c}$ & $86.6 \pm 5.2$ & $68.8 \pm 3.6^{\mathrm{a}}$ & $73.6 \pm 3.5$ & 0.028 \\
\hline Adjusted for age sex and VAT & $88.6 \pm 3.0^{b}$ & $74.0 \pm 2.8^{\mathrm{a}}$ & $73.1 \pm 3.0$ & 0.001 \\
\hline
\end{tabular}

${ }^{a} P<0.05$ between active and remission group; ${ }^{b} P<0.05$ between active group and controls.

fractures with normal or only slightly decreased BMD have been described (1), which can be explained by the qualitative and not the quantitative deterioration of bone tissue. Indeed, GC excess is known to induce alterations in trabecular microarchitecture rendering the trabecular bone specifically more fragile (24). BMF could be a marker of bone quality, more sensitive than BMD to predict risk fracture in CS patients either during active disease or after remission. However, long-term prospective studies with fracture evaluation are needed to test this hypothesis.

Patients with an important clinical and biological impact of their hypercortisolism in our cohort also had a more severe accumulation of BMF. Interestingly, we also found a significant correlation between BMF content and markers of severity of hypercortisolism, such as abnormalities in abdominal fat distribution (increased VAT and dSCAT/sSCAT ratio) and certain biological markers, i.e., $\mathrm{HbA}_{1}$ c. However, in subgroup analysis, VAT was significantly correlated with BMF only in the control group, suggesting that in CS patients, VAT is probably not the main driver of marrow adiposity.

Duration of uncontrolled CS, which is a subjective parameter determined by patients, was not correlated with BMF content, nor was the level of UFC. Other groups have found no correlation between BMD and these two parameters (25). Polymorphisms in the GC receptor gene, which may increase or decrease GC sensitivity could explain these results (26). Our study demonstrates a correlation between VAT volumes and BMF content, but the impact of hypercortisolism on BMF was statistically significant even after adjusting for VAT volumes. Studies with normalweight and obese patients have also found a positive association between VAT and BMF content $(9,26)$, whereas another study on anorexia nervosa (12) found a negative correlation between these parameters. It seems that BMF is an independent fat depot, which can be modulated differently than other fat depots depending on the underlying pathologies. We can therefore consider higher VAT volumes as a reflection of severity of hypercortisolism, rather than a potential actor in BMF accumulation.

Increased $\mathrm{BMF}$ content and decreased $\mathrm{BMD}$ in hypercortisolism could be the result of an imbalance between stromal progenitor cell commitment down the osteogenic and adipogenic lineages in bone marrow (27). Peroxisome proliferator-activated receptor-gamma (PPAR- $\gamma$ ), which represses osteogenic transcription factors (runt-related transcription factor 2 and osterix) and stimulates expression of adipogenic genes in bone marrow, was already found to be modulated by hypercortisolism in VAT (28). Glucocorticoids can also dysregulate Wnt/ $\beta$ catenin signaling, which is a key regulator of the two lineages, by stimulating Wnt antagonists (sclerostin and dickkopf-1), as shown in studies conducted in humans and mice $(29,30,31)$.

One strength of our study is that we used the best available method to measure BMF, which is ${ }^{1} \mathrm{H}-\mathrm{MRS}$, as

Table 4 BMD parameters measured by DXA of active and remission groups. Data presented as mean \pm S.E.M.

Lumbar spine BMD $\left(\mathrm{g} / \mathrm{cm}^{2}\right)$
Lumbar spine BMD T-score
Femoral neck BMD $\left(\mathrm{g} / \mathrm{cm}^{2}\right)$
Femoral neck BMD T-score
Total body BMD $\left(\mathrm{g} / \mathrm{cm}^{2}\right)$
Total body BMD T-score

\begin{tabular}{r} 
Active group \\
\hline $1.045 \pm 0.041$ \\
$-1.125 \pm 0.354$ \\
$0.842 \pm 0.033$ \\
$-1.231 \pm 0.276$ \\
$1.091 \pm 0.035$ \\
$-0.187 \pm 0.298$
\end{tabular}

\begin{tabular}{r}
\hline Remission group \\
\hline $1.067 \pm 0.040$ \\
$-0.500 \pm 0.361$ \\
$0.941 \pm 0.040$ \\
$-0.725 \pm 0.284$ \\
$1.104 \pm 0.035$ \\
$-0.069 \pm 0.301$
\end{tabular}

\begin{tabular}{c}
\hline $\boldsymbol{P}$ value \\
\hline 0.720 \\
0.240 \\
0.085 \\
0.218 \\
0.822 \\
0.806 \\
\hline
\end{tabular}


Table 5 Multiple regression analysis including active and remission groups $(n=34)$

\begin{tabular}{l}
\hline Independent variables \\
\hline Dependent variable: L3 BMF \\
VAT \\
Age \\
Sex \\
Dependent variable: Femur BMF \\
Femoral neck BMD \\
Age \\
Sex \\
Dependent variable: L3 BMF \\
Total body BMD \\
Age \\
Sex
\end{tabular}

\begin{tabular}{|c|c|c|}
\hline$R^{2}$ & $\beta$ & $P$ \\
\hline \multicolumn{3}{|c|}{0.38} \\
\hline & 0.37 & 0.030 \\
\hline & 0.42 & 0.012 \\
\hline & 0.10 & 0.512 \\
\hline \multicolumn{3}{|l|}{0.42} \\
\hline & -0.51 & 0.003 \\
\hline & 0.38 & 0.018 \\
\hline & -0.32 & 0.055 \\
\hline \multicolumn{3}{|l|}{0.81} \\
\hline & -0.99 & 0.003 \\
\hline & 0.20 & 0.323 \\
\hline & -0.46 & 0.103 \\
\hline
\end{tabular}

$\beta$, standardized regression coefficient; $R^{2}$, coefficient of determination.

it allows to quantify BMF compared to water quantity with good reliability and reproducibility (32). Our team has a long experience with ${ }^{1} \mathrm{H}-\mathrm{MRS}$ quantification of myocardial, hepatic and pancreatic triglyceride contents $(33,34,35)$. We measured BMF content at two sites: the femoral neck and the corpus of vertebrae L3, which allowed us to study different kinetics of BMF evolution with aging. Indeed, red marrow is gradually being replaced by BMF throughout aging, and it extends from the appendicular skeleton up to the axial skeleton (6). Another strength of our study is that we did not include hypercortisolism with adrenal etiology to eliminate possible confounding from other co-secreted steroids from adrenal glands. Nevertheless, one study found no difference in BMD between the etiologies of hypercortisolism (36). There are several limitations in our study. The major one is the retrospective nature of our study with a small sample size. However, CS is a very rare disease with an incidence of $0.7-2.4$ per million people per year that makes the feasibility of a large-scale prospective analysis not applicable $(37,38)$ given the high-precision techniques used in our center and not available in the majority of the centers dealing with hypercortisolism. The majority of our patients were treated by hydrocortisone, and this treatment is assumed to impact bone turnover and density at supraphysiological doses (39). However, corticotroph deficiency is now considered as the best prognosis marker to consider that the patients are in remission, and it would have been difficult to evaluate patients in remission if they had not been ACTH deficient for a long period of time after surgery. Moreover, Schulz et al. clearly demonstrated that patients substituted with a dose of hydrocortisone of $25.2 \pm 8.2 \mathrm{mg}$ /day (similar to our study) had normal BMD that did not change after
2 years, suggesting that exogenous replacement therapy within this range of hydrocortisone dosing has no significant impact on BMD. The improvement of diabetes or hypertriglyceridemia could also have an impact on BMF after remission of CS. Anti-osteoporotic therapies taken by some of our patients, could also have modified BMF; however, these treatments were evenly distributed in both the active and remission groups. Finally, we could not calculate fat unsaturation, which could have been interesting as saturated lipids could directly influence osteoblasts (40). As other teams have also reported (33, 41 ), we found at 3 Tesla that the spectroscopic peak at $5.3 \mathrm{ppm}$ was sometimes confounded with the water peak and could not be isolated.

In conclusion, our data demonstrated that BMF content is increased by CS at various localizations and did not differ from normal-weight controls after 43 months of remission. Moreover, the degree of BMF accumulation was positively correlated with markers that reflect the biological and clinical impacts of hypercortisolism. We provide the first study evaluating bone marrow adiposity with ${ }^{1} \mathrm{H}-\mathrm{MRS}$ in such patients. It would be interesting to study further the link between BMF and bone microarchitecture in hypercortisolism.

Supplementary data

This is linked to the online version of the paper at https://doi.org/10.1530/ EJE-18-0318

\section{Declaration of interest}

The authors declare that there is no conflict of interest that could be perceived as prejudicing the impartiality of this study.

\section{Funding}

This work was supported by ARARD (Association d'Assistance médicoTechnique à Domicile), Marseille, France.

\section{Author contribution statement}

Conception and design or analysis and interpretation of data, or both: $F$ M, A D, C V, I A, L P, Y L, R F, C C, A D, T B, F C, B G and P C. Drafting of the manuscript or revising it critically for important intellectual content: $F M$, B G, M B, H D, T G, F K, A D, F C and T B. Final approval of the manuscript submitted: all the authors.

\section{Acknowledgements}

The authors thank the Association pour le Développement des Recherches Biologiques et Médicales (ADEREM) and the Association d'Assistance médico-Technique à Domicile (ARARD) for their financial support. They are also grateful to the patients who participated in the study. They also thank the study staff for their help in recruiting patients and analyzing the data. F Castinetti and B Gaborit: authors contributed equally to the work. 


\section{References}

1 Tóth M \& Grossman A. Glucocorticoid-induced osteoporosis: lessons from Cushing's syndrome. Clinical Endocrinology 201379 1-11. (https://doi.org/10.1111/cen.12189)

2 Canalis E, Mazziotti G, Giustina A \& Bilezikian JP. Glucocorticoidinduced osteoporosis: pathophysiology and therapy. Osteoporosis International 200718 1319-1328. (https://doi.org/10.1007/s00198007-0394-0)

3 Brue T \& Castinetti F. The risks of overlooking the diagnosis of secreting pituitary adenomas. Orphanet Journal of Rare Diseases 2016 11 135. (https://doi.org/10.1186/s13023-016-0516-x)

4 Di Somma C, Pivonello R, Loche S, Faggiano A, Klain M, Salvatore M, Lombardi G \& Colao A. Effect of 2 years of cortisol normalization on the impaired bone mass and turnover in adolescent and adult patients with Cushing's disease: a prospective study. Clinical Endocrinology 200358 302-308 (https://doi.org/10.1046/j.13652265.2003.01713.x)

5 Hermus AR, Smals AG, Swinkels LM, Huysmans DA, Pieters GF, Sweep CF, Corstens FH \& Kloppenborg PW. Bone mineral density and bone turnover before and after surgical cure of Cushing's syndrome. Journal of Clinical Endocrinology and Metabolism $1995 \mathbf{8 0}$ 2859-2865. (https://doi.org/10.1210/jcem.80.10.7559865)

6 Hardouin P, Pansini V \& Cortet B. Bone marrow fat. Joint, Bone, Spine 201481 313-319. (https://doi.org/10.1016/j. jbspin.2014.02.013)

7 Griffith JF, Yeung DKW, Antonio GE, Lee FKH, Hong AWL, Wong SYS, Lau EMC \& Leung PC. Vertebral bone mineral density, marrow perfusion, and fat content in healthy men and men with osteoporosis: dynamic contrast-enhanced MR imaging and MR spectroscopy. Radiology 2005236 945-951. (https://doi.org/10.1148/ radiol.2363041425)

8 Schwartz AV, Sigurdsson S, Hue TF, Lang TF, Harris TB, Rosen CJ, Vittinghoff E, Siggeirsdottir K, Sigurdsson G, Oskarsdottir D et al. Vertebral bone marrow fat associated with lower trabecular BMD and prevalent vertebral fracture in older adults. Journal of Clinical Endocrinology and Metabolism 201398 2294-2300. (https://doi. org/10.1210/jc.2012-3949)

9 Bredella MA, Torriani M, Ghomi RH, Thomas BJ, Brick DJ, Gerweck AV, Rosen CJ, Klibanski A \& Miller KK. Vertebral bone marrow fat is positively associated with visceral fat and inversely associated with IGF-1 in obese women. Obesity 201119 49-53. (https://doi.org/10.1038/oby.2010.106)

10 Schafer AL, Li X, Schwartz AV, Tufts LS, Wheeler AL, Grunfeld C, Stewart L, Rogers SJ, Carter JT, Posselt AM et al. Changes in vertebral bone marrow fat and bone mass after gastric bypass surgery: a pilot study. Bone 201574 140-145. (https://doi.org/10.1016/j. bone.2015.01.010)

11 Baum T, Yap SP, Karampinos DC, Nardo L, Kuo D, Burghardt AJ, Masharani UB, Schwartz AV, Li X \& Link TM. Does vertebral bone marrow fat content correlate with abdominal adipose tissue, lumbar spine bone mineral density, and blood biomarkers in women with type 2 diabetes mellitus? Journal of Magnetic Resonance Imaging 2012 35 117-124. (https://doi.org/10.1002/jmri.22757)

12 Bredella MA, Fazeli PK, Miller KK, Misra M, Torriani M, Thomas BJ, Ghomi RH, Rosen CJ \& Klibanski A. Increased bone marrow fat in anorexia nervosa. Journal of Clinical Endocrinology and Metabolism 200994 2129-2136. (https://doi.org/10.1210/jc.2008-2532)

13 Geer EB, Shen W, Strohmayer E, Post KD \& Freda PU. Body composition and cardiovascular risk markers after remission of Cushing's disease: a prospective study using whole-body MRI. Journal of Clinical Endocrinology and Metabolism 201297 1702-1711. (https:// doi.org/10.1210/jc.2011-3123)

14 Paccou J, Hardouin P, Cotten A, Penel G \& Cortet B. The role of bone marrow fat in skeletal health: usefulness and perspectives for clinicians. Journal of Clinical Endocrinology and Metabolism 2015100 3613-3621. (https://doi.org/10.1210/jc.2015-2338)

15 Zhou A, Murillo H, Cusi K \& Peng Q. Comparison of visceral adipose tissue quantification on water suppressed and nonwater-suppressed MRI at 3.0 tesla. Journal of Magnetic Resonance Imaging 201235 1445-1452. (https://doi.org/10.1002/jmri.23582)

16 Hind K, Oldroyd B \& Truscott JG. In vivo precision of the GE Lunar iDXA densitometer for the measurement of total body composition and fat distribution in adults. European Journal of Clinical Nutrition 201165 140-142. (https://doi.org/10.1038/ejcn.2010.190)

17 Kanis JA, Melton LJ, Christiansen C, Johnston CC \& Khaltaev N. The diagnosis of osteoporosis. Journal of Bone and Mineral Research 19949 1137-1141. (https://doi.org/10.1002/jbmr.5650090802)

18 Vande Berg BC, Malghem J, Lecouvet FE, Devogelaer JP, Maldague B \& Houssiau FA. Fat conversion of femoral marrow in glucocorticoid-treated patients: a cross-sectional and longitudinal study with magnetic resonance imaging. Arthritis and Rheumatism 199942 1405-1411. (https://doi.org/10.1002/15290131(199907)42:7<1405::AID-ANR14>3.0.CO;2-W)

19 Mayo-Smith W, Rosenthal DI, Goodsitt MM \& Klibanski A. Intravertebral fat measurement with quantitative CT in patients with Cushing disease and anorexia nervosa. Radiology 1989170 835-838. (https://doi.org/10.1148/radiology.170.3.2916039)

20 Singhal V \& Bredella MA. Marrow adipose tissue imaging in humans. Bone 2018 Epub. (https://doi.org/10.1016/j. bone.2018.01.009)

21 Kristo C, Jemtland R, Ueland T, Godang K \& Bollerslev J. Restoration of the coupling process and normalization of bone mass following successful treatment of endogenous Cushing's syndrome: a prospective, long-term study. European Journal of Endocrinology 2006 154 109-118. (https://doi.org/10.1530/eje.1.02067)

22 Vestergaard P, Lindholm J, Jørgensen JOL, Hagen C, Hoeck HC, Laurberg P, Rejnmark L, Brixen K, Kristensen LØ, Feldt-Rasmussen U et al. Increased risk of osteoporotic fractures in patients with Cushing's syndrome. European Journal of Endocrinology 2002146 51-56 (https://doi.org/10.1530/eje.0.1460051)

23 Van Staa TP, Leufkens HG, Abenhaim L, Zhang B \& Cooper C. Use of oral corticosteroids and risk of fractures. Journal of Bone and Mineral Research 200015 993-1000. (https://doi.org/10.1359/ jbmr.2000.15.6.993)

24 Vinolas H, Grouthier V, Mehsen-Cetre N, Boisson A, Winzenrieth R, Schaeverbeke T, Mesguich C, Bordenave L \& Tabarin A. Assessment of vertebral microarchitecture in overt and mild Cushing's syndrome using trabecular bone score. Clinical Endocrinology 201889 148-154. (https://doi.org/10.1111/cen.13743)

25 Fütő L, Tőke J, Patócs A, Szappanos Á, Varga I, Gláz E, Tulassay Z, Rácz K \& Tóth M. Skeletal differences in bone mineral area and content before and after cure of endogenous Cushing's syndrome. Osteoporosis International 200819 941-949. (https://doi.org/10.1007/ s00198-007-0514-x)

26 Shen W, Chen J, Punyanitya M, Shapses S, Heshka S \& Heymsfield SB. MRI-measured bone marrow adipose tissue is inversely related to DXA-measured bone mineral in Caucasian women. Osteoporosis International 200718 641-647. (https://doi. org/10.1007/s00198-006-0285-9)

27 Georgiou KR, Hui SK \& Xian CJ. Regulatory pathways associated with bone loss and bone marrow adiposity caused by aging, chemotherapy, glucocorticoid therapy and radiotherapy. American Journal of Stem Cells $20121205-224$.

28 Lindroos J, Husa J, Mitterer G, Haschemi A, Rauscher S, Haas R, Gröger M, Loewe R, Kohrgruber N, Schrögendorfer KF et al. Human but not mouse adipogenesis is critically dependent on LMO3. Cell Metabolism 201318 62-74. (https://doi.org/10.1016/j. cmet.2013.05.020)

29 Gifre L, Ruiz-Gaspà S, Monegal A, Nomdedeu B, Filella X, Guañabens N \& Peris P. Effect of glucocorticoid treatment on 
Wnt signalling antagonists (sclerostin and Dkk-1) and their relationship with bone turnover. Bone 201357 272-276. (https://doi. org/10.1016/j.bone.2013.08.016)

30 Beier EE, Sheu TJ, Resseguie EA, Takahata M, Awad HA, CorySlechta DA \& Puzas JE. Sclerostin activity plays a key role in the negative effect of glucocorticoid signaling on osteoblast function in mice. Bone Research 20175 17013. (https://doi.org/10.1038/ boneres.2017.13)

31 Belaya ZE, Grebennikova TA, Melnichenko GA, Nikitin AG, Solodovnikov AG, Brovkina OI, Grigoriev AU, Rozhinskaya LY \& Dedov II. Effects of endogenous hypercortisolism on bone mRNA and microRNA expression in humans. Osteoporosis International 2018 29 211-221. (https://doi.org/10.1007/s00198-017-4241-7)

32 Li X, Kuo D, Schafer AL, Porzig A, Link TM, Black D \& Schwartz AV. Quantification of vertebral bone marrow fat content using 3 tesla MR spectroscopy: reproducibility, vertebral variation, and applications in osteoporosis. Journal of Magnetic Resonance Imaging 201133 974-979. (https://doi.org/10.1002/jmri.22489)

33 Dutour A, Abdesselam I, Ancel P, Kober F, Mrad G, Darmon P, Ronsin O, Pradel V, Lesavre N, Martin JC et al. Exenatide decreases liver fat content and epicardial adipose tissue in patients with obesity and type 2 diabetes: a prospective randomised clinical trial using magnetic resonance imaging and spectroscopy. Diabetes, Obesity and Metabolism 201618 882-891. (https://doi.org/10.1111/dom.12680)

34 Gaborit B, Jacquier A, Kober F, Abdesselam I, Cuisset T, BoulluCiocca S, Emungania O, Alessi MC, Clément K, Bernard M et al. Effects of bariatric surgery on cardiac ectopic fat: lesser decrease in epicardial fat compared to visceral fat loss and no change in myocardial triglyceride content. Journal of the American College of Cardiology 201260 1381-1389. (https://doi.org/10.1016/j. jacc.2012.06.016)

35 Abdesselam I, Dutour A, Kober F, Ancel P, Bege T, Darmon P, Lesavre N, Bernard M \& Gaborit B. Time course of change in ectopic fat stores after bariatric surgery. Journal of the American College of Cardiology 201667 117-119. (https://doi.org/10.1016/j. jacc.2015.10.052)

36 Tauchmanovà L, Pivonello R, Di Somma C, Rossi R, De Martino MC, Camera L, Klain M, Salvatore M, Lombardi G \& Colao A. Bone demineralization and vertebral fractures in endogenous cortisol excess: role of disease etiology and gonadal status. Journal of Clinical Endocrinology and Metabolism 200691 1779-1784. (https://doi. org/10.1210/jc.2005-0582)

37 Lindholm J, Juul S, Jørgensen JOL, Astrup J, Bjerre P, FeldtRasmussen U, Hagen C, Jørgensen J, Kosteljanetz M, Kristensen L et al. Incidence and late prognosis of Cushing's syndrome: a population-based Study 1. Journal of Clinical Endocrinology and Metabolism 200186 117-123 (https://doi.org/10.1210/ jcem.86.1.7093)

38 Pivonello R, Isidori AM, De Martino MC, Newell-Price J, Biller BMK $\&$ Colao A. Complications of Cushing's syndrome: state of the art. Lancet. Diabetes and Endocrinology 20164 611-629. (https://doi. org/10.1016/S2213-8587(16)00086-3)

39 Schulz J, Frey KR, Cooper MS, Zopf K, Ventz M, Diederich S \& Quinkler M. Reduction in daily hydrocortisone dose improves bone health in primary adrenal insufficiency. European Journal of Endocrinology 2016174 531-538. (https://doi.org/10.1530/EJE-151096)

40 Philippe C, Wauquier F, Landrier JF, Bonnet L, Miot-Noirault E, Rochefort GY, Sadoine J, Asrih M, Jornayvaz FR, Bernalier A et al. GPR40 mediates potential positive effects of a saturated fatty acid enriched diet on bone. Molecular Nutrition and Food Research 2017 61 1600219. (https://doi.org/10.1002/mnfr.201600219)

41 Liney GP, Bernard CP, Manton DJ, Turnbull LW \& Langton CM. Age, gender, and skeletal variation in bone marrow composition: a preliminary study at 3.0Tesla. Journal of Magnetic Resonance Imaging 200726 787-793. (https://doi.org/10.1002/jmri.21072)

Received 13 April 2018

Revised version received 10 August 2018

Accepted 14 August 2018 